\title{
The Association Between Continuous Glucose Monitoring-derived Metrics, Including Time in Range, and Cardiovascular Autonomic Neuropathy in Outpatients With Type 2 Diabetes
}

\section{Min Young Kim}

Samsung Medical Center

Gyuri Kim

Samsung Medical Center

Ji Yun Park

Samsung Medical Center

Min Sun Choi

Samsung Medical Center

Ji Eun Jun

Kyung Hee University Hospital at Gangdong

\section{You-Bin Lee}

Samsung Medical Center

\section{Sang-Man Jin}

Samsung Medical Center

Kyu Yeon Hur

Samsung Medical Center

Jae Hyeon Kim ( $\sim$ jaehyeon@skku.edu )

Samsung Medical Center https://orcid.org/0000-0001-5001-963X

\section{Original investigation}

Keywords: Time in range, type 2 diabetes, cardiovascular autonomic neuropathy, continuous glucose monitoring

Posted Date: November 5th, 2020

DOI: https://doi.org/10.21203/rs.3.rs-101449/v1

License: (9) (1) This work is licensed under a Creative Commons Attribution 4.0 International License. Read Full License 


\section{Abstract}

\section{Background}

Continuous glucose monitoring (CGM)-derived metrics including time in range (TIR) are attracting attention as new indicators of glycemic control and diabetes complications beyond hemoglobin A1c. This study investigated the association between CGM-derived TIR, hyperglycemia, hypoglycemia metrics, and cardiovascular autonomic neuropathy (CAN) in patients with type 2 diabetes.

\section{Methods}

A total of 284 patients with type 2 diabetes who underwent CGM for three days and autonomic function tests within three months based on outpatient data were recruited. The definition of CGM-derived metrics was subject to the most recent international consensus. CAN was defined as an abnormal case in two or more parasympathetic and the severity of CAN was estimated as the sum of the scores of the five cardiovascular autonomic function tests.

Results

Multiple logistic regression analysis revealed that the odds ratio of definite CAN was $0.876[95 \%$ confidence interval (Cl): $0.79-0.98$ ] per $10 \%$ increase in the TIR of 70 to $180 \mathrm{mg} / \mathrm{dL}$, after adjusting for age, sex, diabetes duration, any medications, and glycemic variability. A 10\% increase in TIR was significantly inversely associated with the presence of advanced CAN (OR: $0.89,95 \%$ Cl: $0.81-0.98)$. In addition, there was a strong inverse association between a 10\% increase in the TIR and the total CAN score ( $p$ for trend $=0.001$ ). Among the metrics of hyperglycemia, a time above range $(T A R)$ of greater than $180 \mathrm{mg} / \mathrm{dL}$ was also independently correlated with the presence of definite CAN (OR: $1.013,95 \% \mathrm{Cl}$ : 1.00-1.02) and advanced CAN (OR: 1.01, 95\% Cl: 1.00-1.02).

\section{Conclusions}

A TIR value of 70 to $180 \mathrm{mg} / \mathrm{dL}$ and a TAR value of greater than $180 \mathrm{mg} / \mathrm{dL}$ were significantly associated with cardiovascular autonomic neuropathy in outpatients with type 2 diabetes.

\section{Introduction}

Cardiovascular autonomic neuropathy (CAN) caused by damage to autonomic nerve fibers that innervate the heart and blood vessels is one of the most serious complications of diabetes, leading to abnormal heart-rate control and vascular dynamics dysfunction [1]. In addition, since CAN is an independent predictor of major cardiovascular events, including silent myocardial infarction, and reduces the life expectancy of patients with diabetes, it is important to prevent and recognize CAN promptly in the clinical setting [2-5]. 
As evidence of advanced glucose monitoring options for diabetes treatment has been on the rise recently, studies are emerging that chronic complications of diabetes are linked to glycemic variability and hypoglycemia as well as chronic hyperglycemia. In this sense, continuous glucose monitoring (CGM) overcomes several limitations of existing intermittent measurement methods such as hemoglobin A1c (HbA1c) or self-monitoring glucose monitoring (SMBG), and can provide not only data on the mean glucose level and glycemic variability but can also determine the percent of time spent in hypoglycemia, hyperglycemia, and the target range [6-9].

Among CGM-derived metrics, time in range (TIR) is the simplest and most intuitive key indicator of the quality of glucose control for use throughout the day beyond $\mathrm{HbA} 1 \mathrm{c}$ and has been proposed as an internationally standardized CGM parameter for glycemic targeting in the clinical setting [10]. Several studies have shown to date that TIR and other key parameters are correlated with the clinical outcomes of type 2 diabetes. According to the results of previous studies in patients with type 2 diabetes, TIR has been significantly linked to a lower presence of more advanced diabetic retinopathy and each $10 \%$ increase in the TIR of 70 to $180 \mathrm{mg} / \mathrm{dL}$ was associated with a reduced risk of abnormal carotid intimamedia thickness and albuminuria by $6.4 \%$ and $6.0 \%$, respectively [11-13].

Meanwhile, CAN has been reported to be associated with glucose variability as measured by CGM as well as hypoglycemia in patients with type 1 diabetes $[14,15]$. Even in cross-sectional studies involving patients with type 2 diabetes, it was reported that glycemic variability measured by blinded CGM was associated with heart rate variability, which is one of the earliest indicators of CAN, and the presence of CAN $[16,17]$.

However, no study has yet assessed the association between CAN and CGM metrics discerned by professional CGM in the blinded mode in patients with type 2 diabetes in an outpatient setting, offering real-world clinical data. Therefore, this study will describe the relationship between CGM metrics and CAN in outpatients with type 2 diabetes.

\section{Methods}

\section{Study population}

The patients included in this study were those who underwent CGM and autonomic function testing at the same time or within three months at the Division of Endocrinology and Metabolism of Samsung Medical Center, Seoul, Republic of Korea from March 2009 to July 2019.

Patients with type 1 or gestational diabetes mellitus $(n=108)$; cancer undergoing treatment $(n=5)$; history of thyroid dysfunction $(n=17)$; history of myocardial infarction, revascularization, or stroke $(n=$ 39); severe liver disease defined by a Child-Pugh score of greater than seven points $(n=5)$; estimated glomerular filtration rate (GFR) of less than $30 \mathrm{~mL} / \mathrm{min} / 1.73 \mathrm{~m}^{2}$ derived from the Chronic Kidney Disease Epidemiology Collaboration (CKD-EPI) formula; or lack of relevant clinical data such as HbA1c $(n=2)$ were excluded (Fig. 1). Finally, a total of 284 patients were included in this study. 
The protocol of this study was approved by the institutional review board of Samsung Medical Center (no. 2020-03-111-001).

\section{Clinical variables and biochemical measurements}

Anthropometric measurements such as the height and weight of study participants were collected and the body mass index $\left(\mathrm{kg} / \mathrm{m}^{2}\right)$ was calculated as a result. Systolic and diastolic blood pressure values were recorded as the mean of two blood pressure values measured with the patient remaining in a stable sitting position for five minutes or longer. At the time of CGM, blood samples were collected for biochemical measurements including $\mathrm{HbA1c}$, total cholesterol, high- and low-density lipoprotein cholesterol, triglycerides, C-peptide, and creatinine after a 12-hour overnight fast.

Data on medical history and the use of antihypertensive medications such as angiotensin-converting enzyme inhibitors, angiotensin receptor blockers, and beta-blockers were collected. In addition, lipidlowering agents and antidiabetic agents in use at the time of CGM implementation were reviewed. Hypertension was defined as having a blood pressure of $140 / 90 \mathrm{mmHg}$ or higher or by the current use of antihypertensive medications.

\section{CGM metrics}

The CGM was conducted in a blind manner by using the COLD ${ }^{\mathrm{TM}}$ system (Medtronic MiniMed, North Ridge, CA, USA) for three consecutive days and the collected data were retrospectively reviewed. Study participants calibrated the CGM by measuring capillary blood glucose levels at least twice a day from the first day of CGM implementation. In accordance with the international consensus recommendations for CGM metrics, TIR was defined as the percentage of time spent in the target range of 70 to $180 \mathrm{mg} / \mathrm{dL}$ for the blood glucose level [18]. CGM metrics of hyperglycemia [time above range (TAR) $>250 \mathrm{mg} / \mathrm{dL}$ and $>$ $180 \mathrm{mg} / \mathrm{dL}$ ] hypoglycemia [time below range (TBR) $<70 \mathrm{mg} / \mathrm{dL}$ and $<54 \mathrm{mg} / \mathrm{dL}$ ], and glycemic variability [standard deviation (SD), coefficient of variation (CV)] were also calculated.

\section{Assessment of cardiovascular autonomic neuropathy}

CAN was evaluated as the result of five cardiovascular autonomic function tests proposed by Ewing et al. [19] Patients were asked to avoid vigorous physical activity, smoking, and drinking for 24 hours before the test and to stop consuming coffee and food for three hours before the test. Drugs such as antihistamines, antidepressants, beta-blockers, acetaminophen, and diuretics, which may affect the test results, were stopped 12 hours before the test.

Assessments of the heart rate response to deep breathing (exhalation:inhalation ratio), to standing (30:15 ratio), and to the Valsalva maneuver (Valsalva ratio) were performed to evaluate parasympathetic functions. Two other tests performed to evaluate sympathetic functions included blood pressure responses to standing and to a sustained handgrip, respectively. The heart rate response was assessed 
automatically from electrocardiography recordings using the DICAN evaluation system (Medicore Co., Ltd., Seoul, Korea).

According to the age-specific reference, each of the three parasympathetic function tests was scored as zero points for normal or one point for abnormal results, while each of the two sympathetic function tests was scored as zero points for a normal result, 0.5 points for a borderline result, or one point for an abnormal result [16].

CAN was defined as an abnormal result in two or more parasympathetic tests and the severity of CAN was evaluated as the sum of the partial scores of the five cardiovascular autonomic function tests and categorized as either zero to 1.5 points, two to 2.5 points, and three to five points.

\section{Statistical analysis}

Continuous variables were expressed as mean (SD) values and categorical variables were expressed as ratio or percentages. In the comparison of the baseline characteristics according to the presence or absence of CAN, the Student's $t$-test or the Mann-Whitney $U$ test was used to discern the mean of continuous variables, while the chi-square test was performed for the categorical variables.

Multiple logistic regression analyses were performed to assess the odds ratio (OR) and $95 \%$ confidence interval (Cl) for the association with the presence of CAN with a $10 \%$ increase in the TIR of 70 to 180 $\mathrm{mg} / \mathrm{dL}$, TAR ( $>180 \mathrm{mg} / \mathrm{dl},>250 \mathrm{mg} / \mathrm{dL})$, and TBR ( $<54 \mathrm{mg} / \mathrm{dL},<70 \mathrm{mg} / \mathrm{dL})$, after adjusting for confounding variables such as age, sex, duration of diabetes, and other known risk factors of CAN.

We also conducted subgroup analyses to evaluate the interaction between subgroups regarding age ( $<65$ vs. $\geq 65$ ), duration of diabetes ( $\leq 10$ years vs. $>10$ years), glucose status ( $\mathrm{HbA} 1 \mathrm{c} 7.5 \%$ vs. $>7.5 \%$ ), and the use of insulin or medications that can cause hypoglycemia. In addition, multivariate ordered logistic regression analysis was used to evaluate the association between the severity of CAN and CGM parameters and a trend test across ordered groups was used to evaluate the trend of TIR of 70 to 180 $\mathrm{mg} / \mathrm{dL}$ according to the total CAN score.

Statistical analysis was executed using the Statistical Package for the Social Sciences version 25.0 (IBM Corporation, Armonk, NY, USA) and Stata version 16.0 (StataCorp LLC, College Station, TX, USA), and a $p$ value of less than 0.05 was considered to be statistically significant in two-tailed tests.

\section{Results}

\section{Baseline characteristics}

The baseline characteristics of the enrolled study participants according to the presence of CAN are shown in Table 1. Among the total study population, the mean age was $57.4 \pm 10.5$ years and $58.5 \%$ were men. The mean diabetes duration was $12.0 \pm 8.5$ years and the mean $\mathrm{HbA} 1 \mathrm{c}$ level was $8.3 \% \pm 1.4 \%$. Patients with CAN tended to be younger, with higher HbA1c concentrations; higher diastolic blood 
pressure values; and higher levels of total cholesterol, triglycerides, and low-density lipoprotein cholesterol relative to in study participants without CAN. There was no difference between the two groups in terms of the proportions of use of different medications except angiotensin-converting enzyme inhibitors/angiotensin receptor blockers and lipid-lowering agents.

The mean CGM time was $77.0 \pm 23.7$ hours among patients without CAN and $76.9 \pm 19.2$ hours among study participants with CAN, which were comparable $(p=0.782)$. In study participants with CAN, the mean value when the TAR was greater than $180 \mathrm{mg} / \mathrm{dL}$ was significantly higher (34.4 \pm 27.3 vs. $41.1 \pm$ 27.6; $p=0.049$ ) than that among those without CAN, while other CGM metrics did not show significant differences between the two groups.

\section{CGM target achievement according to the presence of CAN}

The rates of achieving the CGM targets according to the presence of CAN are shown in Figure 2. The targets were selected according to the recent international consensus in the subjects under the age of 65 years. When compared with study participants without CAN, those with CAN had markedly lower rates of achieving targets of TIR of 70 to $180 \mathrm{mg} / \mathrm{dL} \geq 70 \%$ and TAR of $180 \mathrm{mg} / \mathrm{dL}<25 \%$. However, other CGM metrics including the $\mathrm{CV}$ were comparable between the two groups.

\section{Associations between TIR of 70 to $180 \mathrm{mg} / \mathrm{dL}$ and cardiovascular autonomic neuropathy}

To investigate the relationship between TIR of 70 to $180 \mathrm{mg} / \mathrm{dL}$ and CAN, multivariate logistic regression analyses were conducted. As shown in Table 2, the OR for the presence of definite CAN was 0.894 (95\% Cl: $0.81-0.99)$ per $10 \%$ increase TIR of 70 to $180 \mathrm{mg} / \mathrm{dL}$ in the model adjusted for age, sex, and duration of diabetes (Model 1). The association was still significant after further adjustment for the CV as a glycemic variability metric and the use of insulin, oral hypoglycemic agents, aspirin, lipid-lowering agents, and beta-blockers (Model 4) (OR: 0.876, 95\% Cl: 0.79-0.98). However, the correlation between TIR and CAN was not significant in the model adjusted for HbA1c (data not shown). In the subgroup analyses, subjects younger than 65 years, those with diabetes for more than 10 years, those with an $\mathrm{HbA} 1 \mathrm{c}$ concentration of less than $7.5 \%$, and those receiving antidiabetic drugs that could cause hypoglycemia showed a strong inverse association between TIR and CAN, but no interaction was observed (all $p$ for interaction >0.05).

\section{Associations between other core CGM parameters and cardiovascular autonomic neuropathy}

Table 3 shows the relationship between CAN and other CGM core metrics, TAR, and TBR. Hyperglycemia parameters increased the risk of CAN, but only TAR of greater than $180 \mathrm{mg} / \mathrm{dL}$ was statistically significant after adjustment for confounding factors. However, in relation to hypoglycemia, both TBR of less than $70 \mathrm{mg} / \mathrm{dL}$ and TBR of less than $54 \mathrm{mg} / \mathrm{dL}$ were not statistically significant in the adjusted models.

\section{Associations between CGM parameters and the severity of CAN}


Furthermore, we explored the relationship between the severity of CAN assessed by total CAN score and CGM parameters by ordered logistic regression analysis (Table 4). As previously mentioned, the total CAN score was categorized as zero to 1.5 points $(n=171)$, two to 2.5 points $(n=82)$, and three to five points ( = 31). Higher TIR of 70 to $180 \mathrm{mg} / \mathrm{dL}$ significantly lowered the association with the presence of advanced CAN (Model 2) (OR: 0.89, 95\% Cl: 0.81-0.98). Other CGM parameters were not statistically significant, but TAR of greater than $180 \mathrm{mg} / \mathrm{dL}$, among the indicators of hyperglycemia, markedly increased the odds of advanced CAN (Model 2) (OR: 1.01, 95\% Cl: 1.00-1.02). The relationship between the total CAN score and TIR of 70 to $180 \mathrm{mg} / \mathrm{dL}$ is shown in Figure 3; as the total CAN score increased, the TIR of 70 to 180 $\mathrm{mg} / \mathrm{dL}$ tended to decrease in a statistically significant manner ( $p$ for trend $=0.001$ ).

\section{Discussion}

In this study, we firstly investigated whether there was a significant inverse association between CGMderived TIR of 70 to $180 \mathrm{mg} / \mathrm{dL}$ and the presence of cardiovascular autonomic neuropathy in outpatients with type 2 diabetes. This association was maintained even after adjusting for various confounding factors, including the $\mathrm{CV}$ for glycemic variability metrics or the use of beta-blockers, which are known to possibly affect CAN. Additionally, TAR of greater than $180 \mathrm{mg} / \mathrm{dL}$ as a hyperglycemic metric was also independently correlated with CAN, unlike indicators of hypoglycemia.

$\mathrm{HbA1c}$ is heretofore the most well-established tool by which to evaluate glycemic control and treatment efficacy and it is known that improvements in $\mathrm{HbA} 1 \mathrm{c}$ significantly decrease the development of diabetic complications, regardless of whether the patient has type 1 diabetes or type 2 diabetes [20,21]. Several studies have focused on the relationship between $\mathrm{HbA} 1 \mathrm{c}$ and metrics that can be measured using CGM. Vigersky et al. [22] analyzed 18 randomized controlled trials including 2577 patients with type 1 diabetes and type 2 diabetes and reported a strong correlation between $\mathrm{HbA} 1 \mathrm{c}$ and TIR of 70 to $180 \mathrm{mgl} / \mathrm{dL}$, while Beck et al. [23] found that a $10 \%$ increase in TIR of 70 to $180 \mathrm{mg} / \mathrm{dL}$ decreased the mean $\mathrm{A} 1 \mathrm{c}$ by $0.6 \%$.

Given the above correlation between TIR and HbA1c, TIR could be a useful surrogate marker for various diabetes complications, with several previous studies supporting this idea. Lu et al. reported that, in the relationship between TIR and diabetic retinopathy in patients with type 2 diabetes, the TIR quartile and the severity of diabetic retinopathy were inversely correlated [11]. In addition, in the relationship between TIR and carotid intima-media thickness, which is a surrogate marker for cardiovascular disease, when TIR was increased by $10 \%$ the risk of abnormal carotid intima-media thickness decreased by $6.4 \%$ [12]. We previously reported that albuminuria, a microvascular complication of diabetes, was found to be associated with TIR and hyperglycemic metrics and, as in other studies, it was inversely correlated even after adjusting the glycemic variability metrics [13]. The results of these previous studies strengthen the potential of TIR as a tool to predict the risk of complications of diabetes.

Recently, Guo et al. reported that TIR is inversely correlated with the presence of CAN independent of $\mathrm{HbA1c}$ and glycemic variability metrics in hospitalized patients with type 2 diabetes using CGM [24]. However, the hospitalized state is subject to changes in dietary pattern, physical activity, and compliance 
with medication, which are important factors in glycemic control in patients with diabetes and may not reflect the patient's true daily lifestyle. In line with this, there was a discrepancy between the assumed TIR from baseline $\mathrm{HbA} 1 \mathrm{c}$ and the real TIR, which was relatively better than that which was assumed, in the hospitalized setting. However, in the present study, as we retrospectively confirmed blood glucose levels using blind CGM and the actual life pattern of the patients could be reflected in the outpatient setting, there was a reduced discrepancy between the value of $\mathrm{HbA1 \textrm {c }}$ at baseline and TIR. In this manner, probably due to the strong association between $\mathrm{HbA} 1 \mathrm{c}$ at baseline and TIR, our study did not show a significant association between TIR and CAN after further adjusting for HbA1c (data not shown), which was similar to in our previous study concerning the relationship between TIR and albuminuria in the outpatient setting [13].

In addition, this study showed that TAR of greater than $180 \mathrm{mg} / \mathrm{dL}$ was independently associated with the presence of CAN even after adjusting the CV as a glycemic variability metric. Although the mechanism of diabetic CAN is still not fully understood, known risk factors include chronic hyperglycemia, obesity, hypertriglyceridemia, and duration of diabetes. Among these, hyperglycemia plays a pivotal role in the initial pathophysiology and progression of CAN by inducing direct neuronal damage or dysfunction with increased oxidative and nitrosative stress and by inducing endothelial dysfunction that leads to neuronal ischemia [25-27].

The strength of this study is that it is the first report to assess the relationship between CAN and CGM metrics including hyperglycemic and hypoglycemic metrics as well as TIR of target glucose, which were collected by blind manner, in outpatients with type 2 diabetes. In addition, we adopted a recently updated international consensus report for evaluating the proportion of patients who met the glycemic target of TIR and other CGM-derived metrics. Moreover, we evaluated the presence of CAN as well as the severity of CAN using the total CAN score and confirmed the inverse relationship between TIR and CAN. Types of antidiabetic agents able to affect TIR or CAN were examined and adjusted for in the analyses.

However, this study also has several limitations that should be noted. The sample size in this study was small, and since it was conducted on outpatients and with people who need CGM, its retrospective nature that is sensitive to selection bias requires careful estimation when applying the results in different groups. Second, CGM data were only obtained for three days and this time may be too short of a duration in which to capture a complete glycemic pattern. Third, this study was designed as a cross-sectional investigation so it is difficult to estimate the causal relationship between exposure and outcome. Further longitudinal studies are needed.

\section{Conclusions}

This study supports that TIR of 70 to $180 \mathrm{mg} / \mathrm{dL}$ as measured by CGM is independently associated with the presence and severity of CAN in patients with type 2 diabetes patients seen on an outpatient basis. TAR of greater than $180 \mathrm{mg} / \mathrm{dL}$ as a hyperglycemic metric was also independently associated with the presence of CAN. 


\section{Abbreviations}

CAN: Cardiovascular autonomic neuropathy; CGM: Continuous glucose monitoring; Cl: Confidence intervals; CV: Coefficient of variation; GFR: Glomerular filtration rate; HbA1c: Hemoglobin A1c; OR: Odds ratio; SD: Standard deviation; SMBG: Self-monitoring glucose monitoring; TAR: Time above range; TBR: Time below range; TIR: Time in range.

\section{Declarations}

\section{Acknowledgments}

Not applicable

\section{Funding}

This research was supported by a grant of the Korea Health Technology R\&D Project through the Korea Health Industry Development Institute (KHIDI), funded by the Ministry of Health \& Welfare, Republic of Korea (grant number : HI19C0543). This research was funded by the Korea Disease Control and Prevention Agency, grant number 2020-ER6402-00.

\section{Ethics approval and consent to participate}

This study was approved by the institutional review board of Samsung Medical Center (no. 2020-03-111001).

\section{Consent for publication}

Not applicable

\section{Availability of data and materials}

The datasets generated and/or analyzed during the current study are available from the corresponding authors on reasonable request.

\section{Competing interests}

The authors declare that they have no competing interests.

\section{Authors' contributions}

MYK, JHK and GK are responsible for this study. GK and JHK conceived and designed this study. MYK, JYP, MSC, KYH, S-MJ, Y-BL and JEJ collected data. MYK conducted statistical analyses and drafted the manuscript. GK and JHK reviewed and revised the manuscript. All authors read and approved the final manuscript. 


\section{References}

1. Vinik Al, Erbas T, Casellini CM. Diabetic cardiac autonomic neuropathy, inflammation and cardiovascular disease. J Diabetes Investig. 2013;4(1):4-18.

2. Dimitropoulos G, Tahrani AA, Stevens MJ. Cardiac autonomic neuropathy in patients with diabetes mellitus. World J Diabetes. 2014;5(1):17-39.

3. Chyun DA, Wackers FJ, Inzucchi SE, Jose P, Weiss C, Davey JA, et al. Autonomic dysfunction independently predicts poor cardiovascular outcomes in asymptomatic individuals with type 2 diabetes in the DIAD study. SAGE Open Med. 2015;3:2050312114568476.

4. Ang L, Dillon B, Mizokami-Stout K, Pop-Busui R. Cardiovascular autonomic neuropathy: A silent killer with long reach. Auton Neurosci. 2020;225:102646.

5. Spallone V. Update on the Impact, Diagnosis and Management of Cardiovascular Autonomic Neuropathy in Diabetes: What Is Defined, What Is New, and What Is Unmet. Diabetes Metab J. 2019;43(1):3-30.

6. Wright LA, Hirsch IB. Metrics Beyond Hemoglobin A1C in Diabetes Management: Time in Range, Hypoglycemia, and Other Parameters. Diabetes Technol Ther. 2017;19(S2):S16-S26.

7. Bergenstal RM, Ahmann AJ, Bailey T, Beck RW, Bissen J, Buckingham B, et al. Recommendations for standardizing glucose reporting and analysis to optimize clinical decision making in diabetes: the ambulatory glucose profile. J Diabetes Sci Technol. 2013;7(2):562-78.

8. Danne T, Nimri R, Battelino T, Bergenstal RM, Close KL, DeVries JH, et al. International Consensus on Use of Continuous Glucose Monitoring. Diabetes Care. 2017;40(12):1631-40.

9. Jung HS. Clinical Implications of Glucose Variability: Chronic Complications of Diabetes. Endocrinol Metab (Seoul). 2015;30(2):167-74.

10. American Diabetes Association. 6. Glycemic Targets: Standards of Medical Care in Diabetes-2020. Diabetes Care. 2020;43(Suppl 1):S66-S76.

11. Lu J, Ma X, Zhou J, Zhang L, Mo Y, Ying L, et al. Association of Time in Range, as Assessed by Continuous Glucose Monitoring, With Diabetic Retinopathy in Type 2 Diabetes. Diabetes Care. 2018;41(11):2370-6.

12. Lu J, Ma X, Shen Y, Wu Q, Wang R, Zhang L, et al. Time in Range Is Associated with Carotid IntimaMedia Thickness in Type 2 Diabetes. Diabetes Technol Ther. 2020;22(2):72-8.

13. Yoo JH, Choi MS, Ahn J, Park SW, Kim Y, Hur KY, et al. Association Between Continuous Glucose Monitoring-Derived Time in Range, Other Core Metrics, and Albuminuria in Type 2 Diabetes. Diabetes Technol Ther. 2020.

14. Jun JE, Lee SE, Lee YB, Ahn JY, Kim G, Hur KY, et al. Continuous glucose monitoring defined glucose variability is associated with cardiovascular autonomic neuropathy in type 1 diabetes. Diabetes Metab Res Rev. 2019;35(2):e3092.

15. Jaiswal M, McKeon K, Comment N, Henderson J, Swanson S, Plunkett C, et al. Association between impaired cardiovascular autonomic function and hypoglycemia in patients with type 1 diabetes. 
Diabetes Care. 2014;37(9):2616-21.

16. Jun JE, Jin SM, Baek J, Oh S, Hur KY, Lee MS, et al. The association between glycemic variability and diabetic cardiovascular autonomic neuropathy in patients with type 2 diabetes. Cardiovasc Diabetol. 2015;14:70.

17. Kalopita S, Liatis S, Thomakos P, Vlahodimitris I, Stathi C, Katsilambros N, et al. Relationship between autonomic nervous system function and continuous interstitial glucose measurement in patients with type 2 diabetes. J Diabetes Res. 2014;2014:835392.

18. Battelino T, Danne T, Bergenstal RM, Amiel SA, Beck R, Biester T, et al. Clinical Targets for Continuous Glucose Monitoring Data Interpretation: Recommendations From the International Consensus on Time in Range. Diabetes Care. 2019;42(8):1593-603.

19. Ewing DJ, Martyn CN, Young RJ, Clarke BF. The value of cardiovascular autonomic function tests: 10 years experience in diabetes. Diabetes Care. 1985;8(5):491-8.

20. Diabetes Control and Complications Trial (DCCT): results of feasibility study. The DCCT Research Group. Diabetes Care. 1987;10(1):1-19.

21. King P, Peacock I, Donnelly R. The UK prospective diabetes study (UKPDS): clinical and therapeutic implications for type 2 diabetes. Br J Clin Pharmacol. 1999;48(5):643-8.

22. Vigersky RA, McMahon C. The Relationship of Hemoglobin A1C to Time-in-Range in Patients with Diabetes. Diabetes Technol Ther. 2019;21(2):81-5.

23. Beck RW, Bergenstal RM, Cheng P, Kollman C, Carlson AL, Johnson ML, et al. The Relationships Between Time in Range, Hyperglycemia Metrics, and HbA1c. J Diabetes Sci Technol. 2019;13(4):61426.

24. Guo Q, Zang P, Xu S, Song W, Zhang Z, Liu C, et al. Time in Range, as a Novel Metric of Glycemic Control, Is Reversely Associated with Presence of Diabetic Cardiovascular Autonomic Neuropathy Independent of HbA1c in Chinese Type 2 Diabetes. J Diabetes Res. 2020;2020:5817074.

25. Rolim LC, Sa JR, Chacra AR, Dib SA. Diabetic cardiovascular autonomic neuropathy: risk factors, clinical impact and early diagnosis. Arq Bras Cardiol. 2008;90(4):e24-31.

26. Vinik Al, Erbas T. Diabetic autonomic neuropathy. Handb Clin Neurol. 2013;117:279-94.

27. Leinninger GM, Edwards JL, Lipshaw MJ, Feldman EL. Mechanisms of disease: mitochondria as new therapeutic targets in diabetic neuropathy. Nat Clin Pract Neurol. 2006;2(11):620-8.

\section{Tables}

Table 1 Baseline characteristics according to the presence of CAN $(n=284)$ 


\begin{tabular}{|c|c|c|c|}
\hline & $\begin{array}{l}\text { No CAN } \\
(n=200,70.4 \%)\end{array}$ & $\begin{array}{l}\text { CAN } \\
(n=84,29.6 \%)\end{array}$ & $p$ value \\
\hline Age, years & $59.5(10.6)$ & $52.2(8.1)$ & $<0.001$ \\
\hline Sex, $n$ & & & 0.196 \\
\hline Men & $112(56.0)$ & $54(64.3)$ & \\
\hline Women & $88(44.0)$ & $30(35.7)$ & \\
\hline $\mathrm{BMI}, \mathrm{kg} / \mathrm{m}^{2}$ & $24.6(2.9)$ & $25.3(3.8)$ & 0.089 \\
\hline Duration of diabetes, years & $12.6(9.2)$ & $10.8(6.6)$ & 0.076 \\
\hline $\mathrm{HbA} 1 \mathrm{c}, \%$ & $8.1(1.4)$ & $8.5(1.5)$ & 0.041 \\
\hline SBP, mmHg & $124.5(15.6)$ & $126.8(15.6)$ & 0.262 \\
\hline $\mathrm{DBP}, \mathrm{mmHg}$ & $74.4(10.4)$ & $80.1(10.6)$ & $<0.001$ \\
\hline Total cholesterol, mg/dL & $164.7(84.9)$ & $170.3(37.6)$ & 0.562 \\
\hline $\mathrm{HDL}-\mathrm{C}, \mathrm{mg} / \mathrm{dL}$ & $50.5(13.4)$ & $47.9(14.3)$ & 0.145 \\
\hline Triglycerides, mg/dL & $130.5(102.2)$ & $168.5(133.6)$ & 0.021 \\
\hline LDL-C, mg/dL & $89.9(26.7)$ & $100.1(34.7)$ & 0.017 \\
\hline eGFR, mL/min per $1.73 \mathrm{~m}^{2}$ & $83.9(21.5)$ & $88.7(27.5)$ & 0.156 \\
\hline C-peptide, $\mathrm{ng} / \mathrm{mL}$ & $1.9(1.4)$ & $2.2(1.4)$ & 0.098 \\
\hline \multicolumn{4}{|l|}{ Use of anti-diabetic agents } \\
\hline Metformin & $141(70.5)$ & $52(61.9)$ & 0.157 \\
\hline DPP-4 inhibitors & $43(21.5)$ & 15 (17.9) & 0.487 \\
\hline Sulfonylurea & $82(41.0)$ & $40(47.9)$ & 0.304 \\
\hline SGLT-2 inhibitors & $0(0.0)$ & $2(2.3)$ & 0.029 \\
\hline Thiazolidinediones & $9(4.5)$ & $5(6.0)$ & 0.606 \\
\hline Glinides & $14(7.0)$ & $5(6.0)$ & 0.747 \\
\hline a-glucosidase inhibitors & $32(16.0)$ & $14(16.7)$ & 0.889 \\
\hline GLP-1 receptor agonists & $1(0.5)$ & $1(1.2)$ & 0.525 \\
\hline insulin & $97(48.5)$ & $40(47.6)$ & 0.892 \\
\hline Use of lipid-lowering agents & $108(54.0)$ & $33(39.3)$ & 0.024 \\
\hline Use of beta-blocker & $14(7.0)$ & $5(6.0)$ & 0.747 \\
\hline
\end{tabular}




\begin{tabular}{|c|c|c|c|}
\hline Use of ARB or ACEI & $87(43.5)$ & $48(57.1)$ & 0.036 \\
\hline Hypertension & $126(63.0)$ & $56(66.7)$ & 0.557 \\
\hline \multicolumn{4}{|l|}{ CGM metrics } \\
\hline Amount of CGM data, hours & $77.0(23.7)$ & $76.2(18.2)$ & 0.782 \\
\hline \multicolumn{4}{|l|}{ Mean glucose, $\mathrm{mg} / \mathrm{dL}$} \\
\hline Mean (SD) & $168.3(46.2)$ & $176.4(41.9)$ & 0.166 \\
\hline \multicolumn{4}{|l|}{ GMI, \% } \\
\hline Mean (SD) & $7.3(1.1)$ & $7.5(1.0)$ & 0.166 \\
\hline \multicolumn{4}{|l|}{ TIR (70-180 mg/dL), \% } \\
\hline Mean (SD) & $62.7(26.8)$ & $57.0(27.0)$ & 0.105 \\
\hline \multicolumn{4}{|l|}{ TAR $(>180 \mathrm{mg} / \mathrm{dL}), \%$} \\
\hline Mean (SD) & $34.4(27.3)$ & $41.4(27.6)$ & 0.049 \\
\hline \multicolumn{4}{|l|}{ TAR $(>250 \mathrm{mg} / \mathrm{dL}), \%$} \\
\hline Mean (SD) & $13.4(18.6)$ & $14.3(17.0)$ & 0.678 \\
\hline \multicolumn{4}{|l|}{ TBR (<70 mg/dL), \% } \\
\hline Mean (SD) & $2.9(5.6)$ & $1.6(3.7)$ & 0.018 \\
\hline \multicolumn{4}{|l|}{ TBR (<54 mg/dL), \% } \\
\hline Mean (SD) & $1.2(3.7)$ & $0.5(1.4)$ & 0.021 \\
\hline \multicolumn{4}{|l|}{ SD } \\
\hline Mean (SD) & $50.0(21.8)$ & $49.4(19.6)$ & 0.831 \\
\hline \multicolumn{4}{|l|}{$C V, \%$} \\
\hline Mean (SD) & $29.6(10.5)$ & $27.7(8.7)$ & 0.119 \\
\hline
\end{tabular}

$A C E l$ angiotensin-converting enzyme inhibitors, $A R B$ angiotensin receptor blocker, $B M /$ body mass index, $C A N$ cardiovascular autonomic neuropathy, $C G M$ continuous glucose monitoring, $C V$ coefficient of variation, $D B P$ diastolic blood pressure, DPP-4 dipeptidyl peptidase-4, eGFR estimated glomerular filtration rate, GLP-1 glucagon like peptide-1, GMI glucose management indicator, $H D L-C$ high-density lipoprotein cholesterol, $L D L-C$ low-density lipoprotein cholesterol, $S B P$ systolic blood pressure, $S D$ standard deviation, SGLT-2 sodium glucose cotransporter-2, TAR time above range, TBR time below range, TIR time in range 


\begin{tabular}{|llll|}
\hline & Odds ratio (95\% Cl) & $p$ value & \\
\hline Model 1 & $0.894(0.81-0.99)$ & 0.034 & \\
\hline Model 2 & $0.887(0.89-0.99)$ & 0.029 & \\
\hline Model 3 & $0.882(0.79-0.98)$ & 0.023 & \\
\hline Model 4 & $0.876(0.79-0.98)$ & 0.017 & \\
\hline Subgroupt & Odds ratio (95\% Cl) & $p$ value & p for interaction \\
\hline Age & & & 0.221 \\
\hline$<65(n=220)$ & $0.873(0.78-0.98)$ & 0.022 & \\
\hline$\geq 65(n=64)$ & $0.829(0.47-1.47)$ & 0.519 & \\
\hline Duration of diabetes & & & 0.601 \\
\hline$\leq 10$ years $(n=130)$ & $0.950(0.81-1.12)$ & 0.539 & \\
\hline$>10$ years $(n=154)$ & $0.797(0.66-0.96)$ & 0.015 & \\
\hline HbA1c & & & 0.176 \\
\hline$\leq 7.5 \%(n=99)$ & $0.655(0.46-0.93)$ & 0.017 & \\
\hline$>7.5 \%(n=185)$ & $0.936(0.81-1.08)$ & 0.363 & \\
\hline Sulfonylurea or glinide & & & \\
\hline Yes $(n=141)$ & $0.782(0.66-0.93)$ & 0.004 & \\
\hline No $(n=143)$ & $0.981(0.83-1.17)$ & 0.832 & \\
\hline Sulfonylurea or glinide or insulin & & & \\
\hline Yes $(n=227)$ & & & \\
\hline No $(n=57)$ & & & \\
\hline
\end{tabular}

* Odds ratios represent per $10 \%$ increase in TIR

Model 1: adjusted for age, sex and duration of diabetes

Model 2: adjusted for model 1 plus any medication (insulin, OHA, aspirin, dyslipidemia)

Model 3: adjusted for model 2 plus CV

Model 4: adjusted for model 3 plus beta-blocker

† Subgroups were analyzed for model 4 
$C A N$ cardiovascular autonomic neuropathy, $\mathrm{Cl}$ confidence interval, $\mathrm{OHA}$ oral hypoglycemic agent, $T I R$ time in range

Table 3 Association between other CGM parameter and CAN

\begin{tabular}{|c|c|c|c|}
\hline CGM metrics & & Odds ratio $(95 \% \mathrm{Cl})$ & $p$ value \\
\hline \multirow[t]{2}{*}{ TAR $(>250 \mathrm{mg} / \mathrm{dL})$} & Model 1 & $1.005(0.99-1.02)$ & 0.534 \\
\hline & Model 2 & $1.005(0.99-1.02)$ & 0.509 \\
\hline \multirow[t]{2}{*}{ TAR $(>180 \mathrm{mg} / \mathrm{dL})$} & Model 1 & $1.011(1.00-1.02)$ & 0.025 \\
\hline & Model 2 & $1.013(1.00-1.02)$ & 0.016 \\
\hline \multirow[t]{2}{*}{ TBR (<70mg/dL) } & Model 1 & $0.933(0.86-1.01)$ & 0.090 \\
\hline & Model 2 & $0.911(0.82-1.01)$ & 0.071 \\
\hline \multirow[t]{2}{*}{ TBR (<54mg/dL) } & Model 1 & $0.899(0.77-1.05)$ & 0.181 \\
\hline & Model 2 & $0.884(0.73-1.07)$ & 0.196 \\
\hline
\end{tabular}

Model 1: adjusted for age, sex and duration of diabetes

Model 2: adjusted for age, sex, duration of diabetes, any medications (insulin, OHA, aspirin, dyslipidemia), $\mathrm{CV}$, and beta-blocker

$C A N$ cardiovascular autonomic neuropathy, $C G M$ continuous glucose monitoring, $C /$ confidence interval, $C V$ coefficient of variation, $O H A$ oral hypoglycemic agent, TAR time above range, TBR time below range

Table 4 Ordered logistic regression analysis of the associations between CGM parameters and the severity of CAN 


\begin{tabular}{|c|c|c|c|}
\hline CGM metrics & & Odds ratio $(95 \% \mathrm{Cl})$ & $p$ value \\
\hline \multirow[t]{2}{*}{ TIR } & Model 1 & $0.89(0.81-0.97)$ & 0.012 \\
\hline & Model 2 & $0.89(0.81-0.98)$ & 0.023 \\
\hline \multirow[t]{2}{*}{ TAR $(>250 \mathrm{mg} / \mathrm{dL})$} & Model 1 & $1.01(1.00-1.02)$ & 0.186 \\
\hline & Model 2 & $1.01(0.99-1.02)$ & 0.347 \\
\hline \multirow[t]{2}{*}{ TAR $(>180 \mathrm{mg} / \mathrm{dL})$} & Model 1 & $1.01(1.00-1.02)$ & 0.017 \\
\hline & Model 2 & $1.01(1.00-1.02)$ & 0.025 \\
\hline \multirow[t]{2}{*}{ TBR (<70mg/dL) } & Model 1 & $0.98(0.93-1.03)$ & 0.473 \\
\hline & Model 2 & $0.94(0.87-1.01)$ & 0.088 \\
\hline \multirow[t]{2}{*}{ TBR $(<54 \mathrm{mg} / \mathrm{dL})$} & Model 1 & $1.01(0.93-1.09)$ & 0.896 \\
\hline & Model 2 & $0.97(0.88-1.08)$ & 0.627 \\
\hline
\end{tabular}

* CAN scores categorized 0-1.5 points $(n=171), 2-2.5$ points $(n=84)$, and 3-5 points $(n=31)$.

* Odds ratios represent per $10 \%$ increase in TIR

Model 1: adjusted for age, sex and duration of diabetes

Model 2: adjusted for age, sex, duration of diabetes, any medications, CV, and beta-blocker $C A N$ cardiovascular autonomic neuropathy, $C G M$ continuous glucose monitoring, $C /$ confidence interval, $C V$ coefficient of variation, TAR time above range, TBR time below range, TIR time in range

\section{Figures}




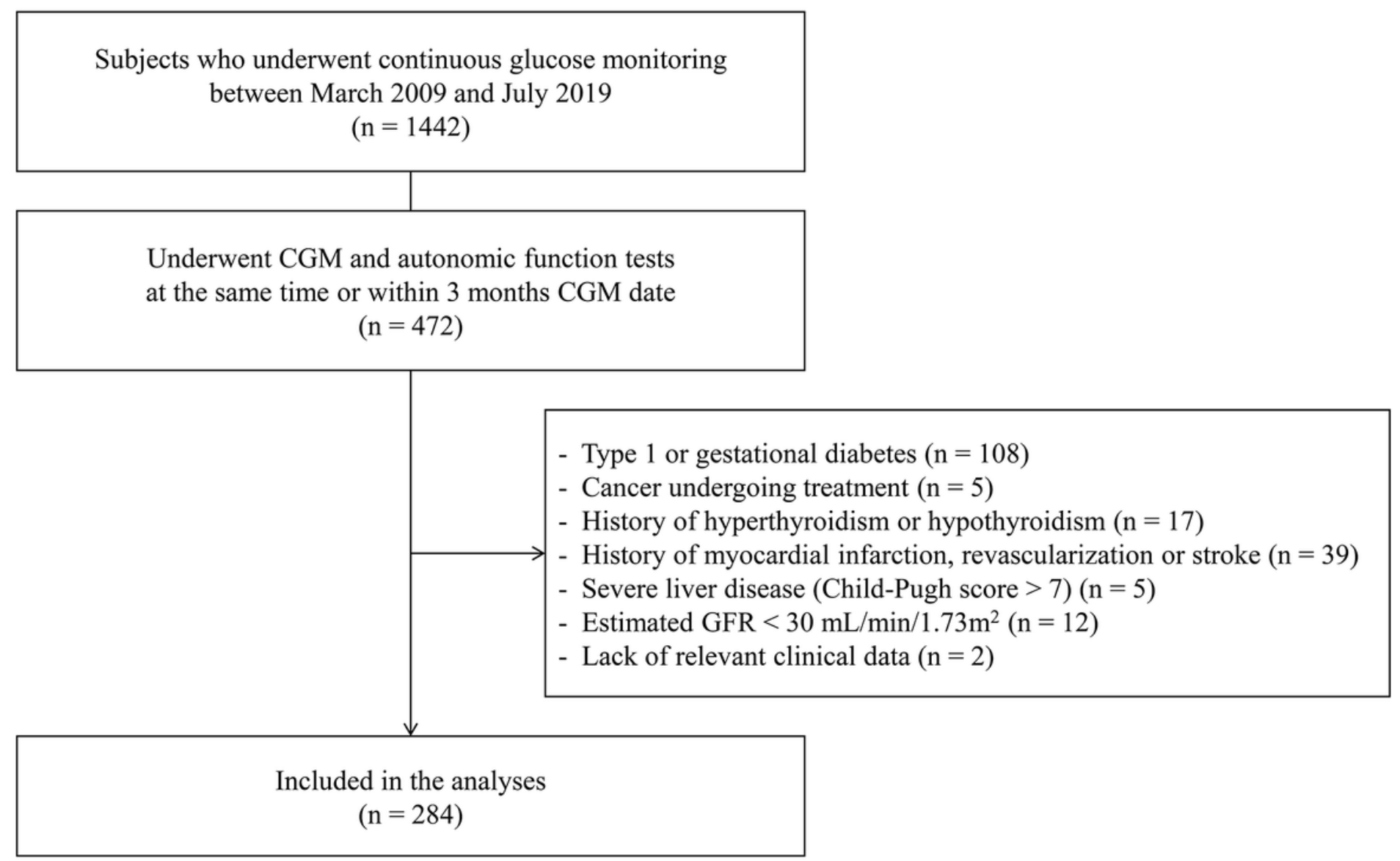

Figure 1

Inclusion and exclusion criteria for the study population CGM continuous glucose monitoring, GFR glomerular filtration rate 


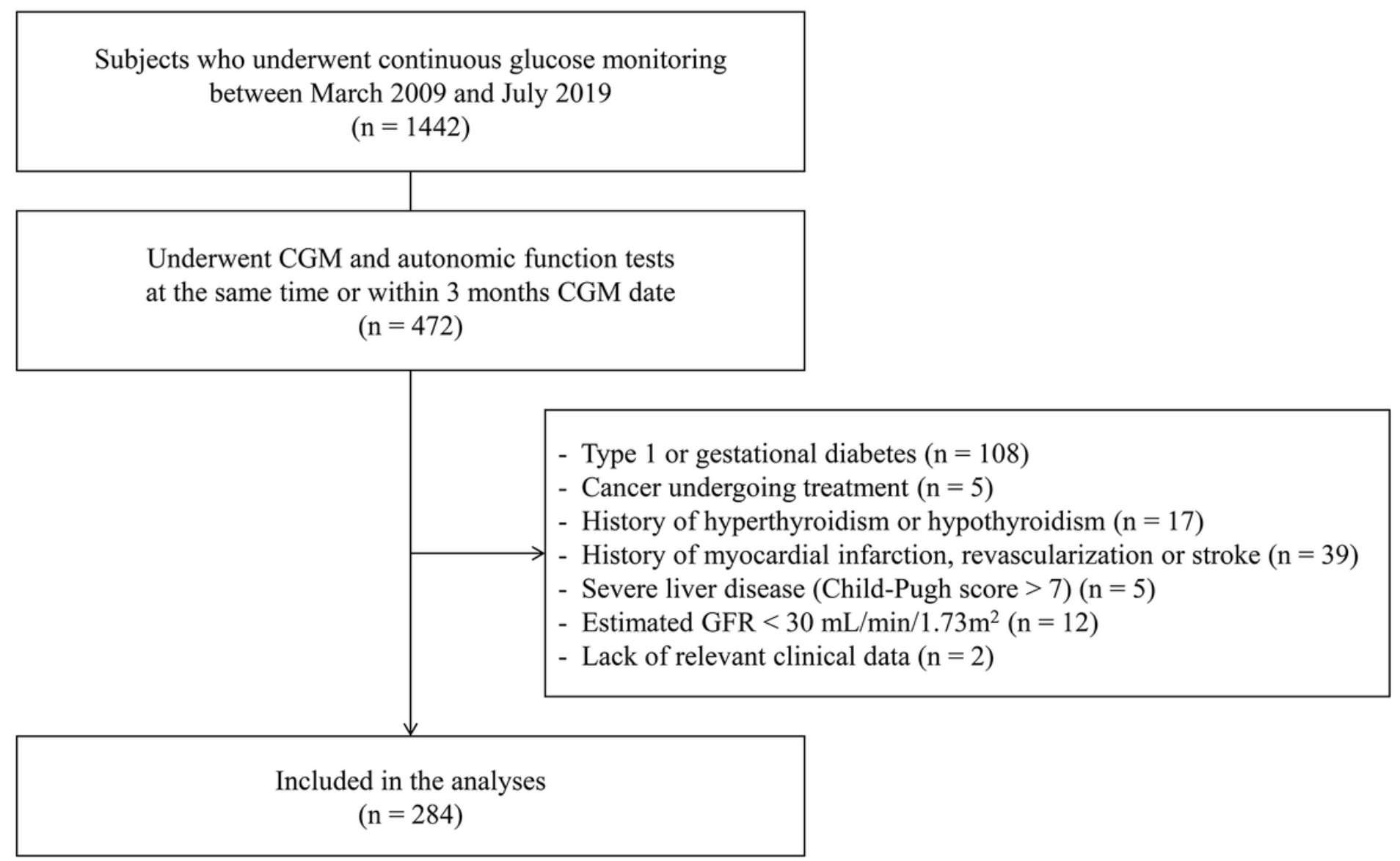

Figure 1

Inclusion and exclusion criteria for the study population CGM continuous glucose monitoring, GFR glomerular filtration rate 


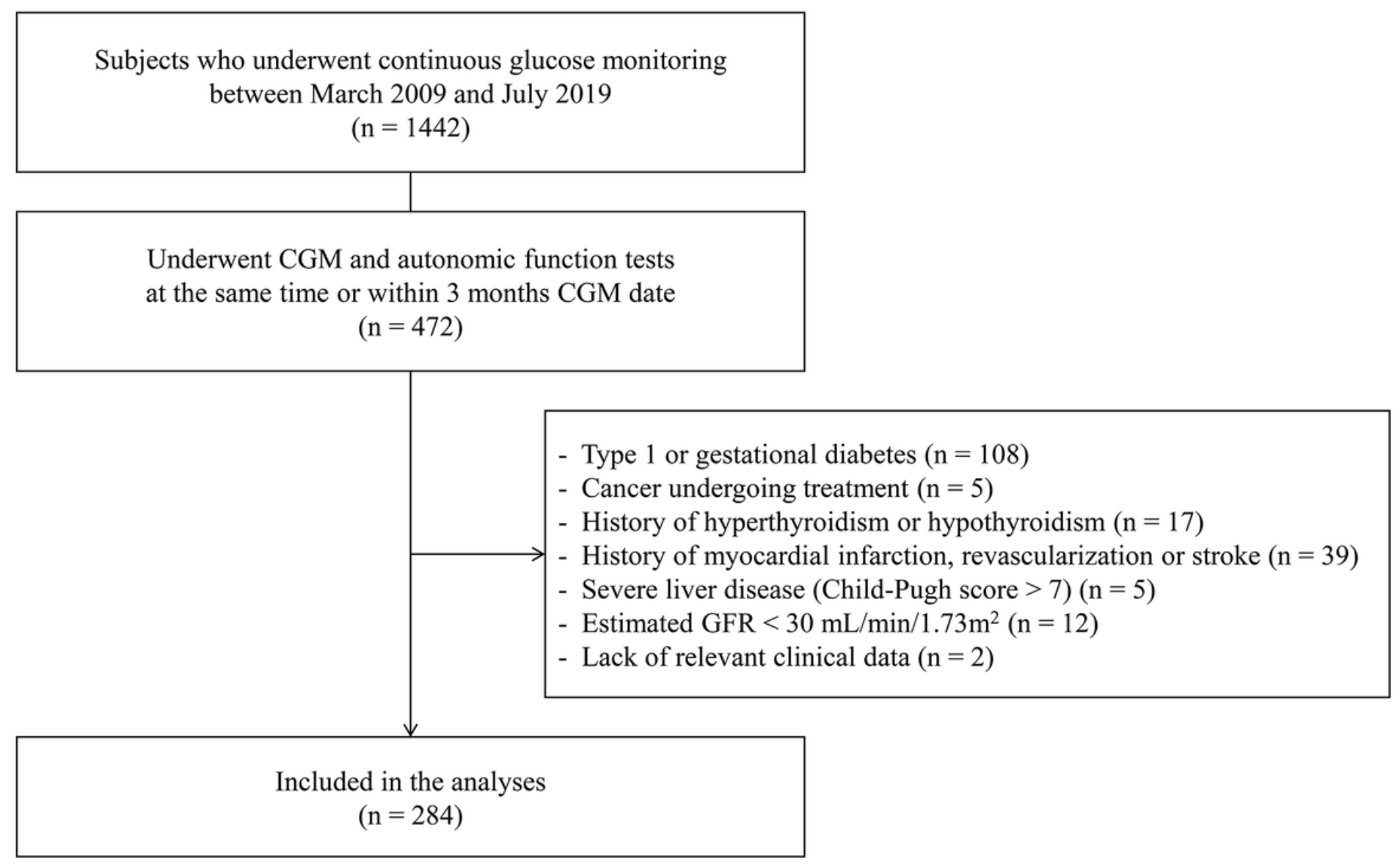

Figure 1

Inclusion and exclusion criteria for the study population CGM continuous glucose monitoring, GFR glomerular filtration rate 

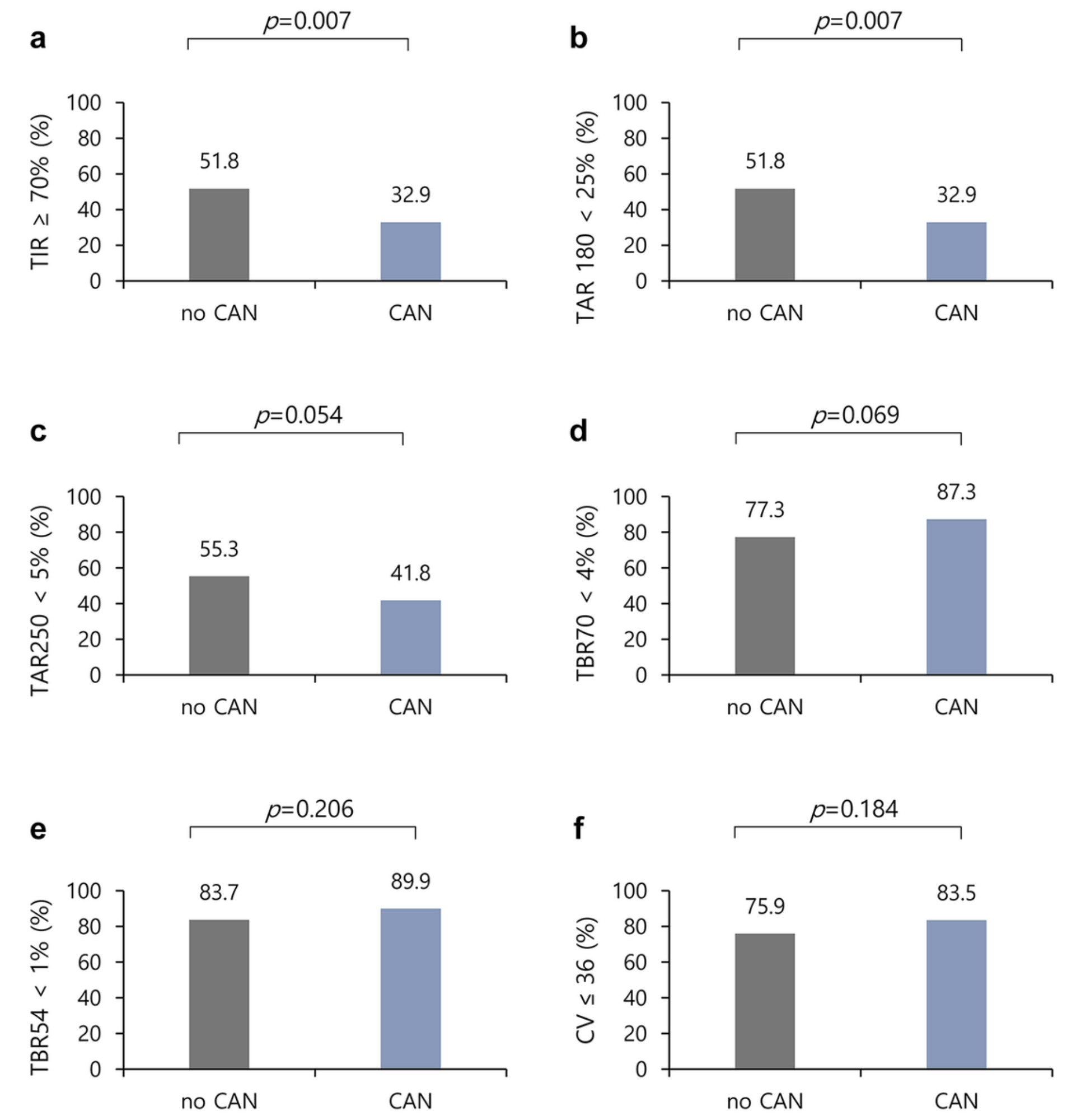

\section{Figure 2}

Prevalence of subjects who achieved the CGM targets according to the presence of CAN in study participants younger than 65 years $(n=220)$ CAN cardiovascular autonomic neuropathy, CGM continuous glucose monitoring, TAR time above range, TBR time below range, TIR time in range 

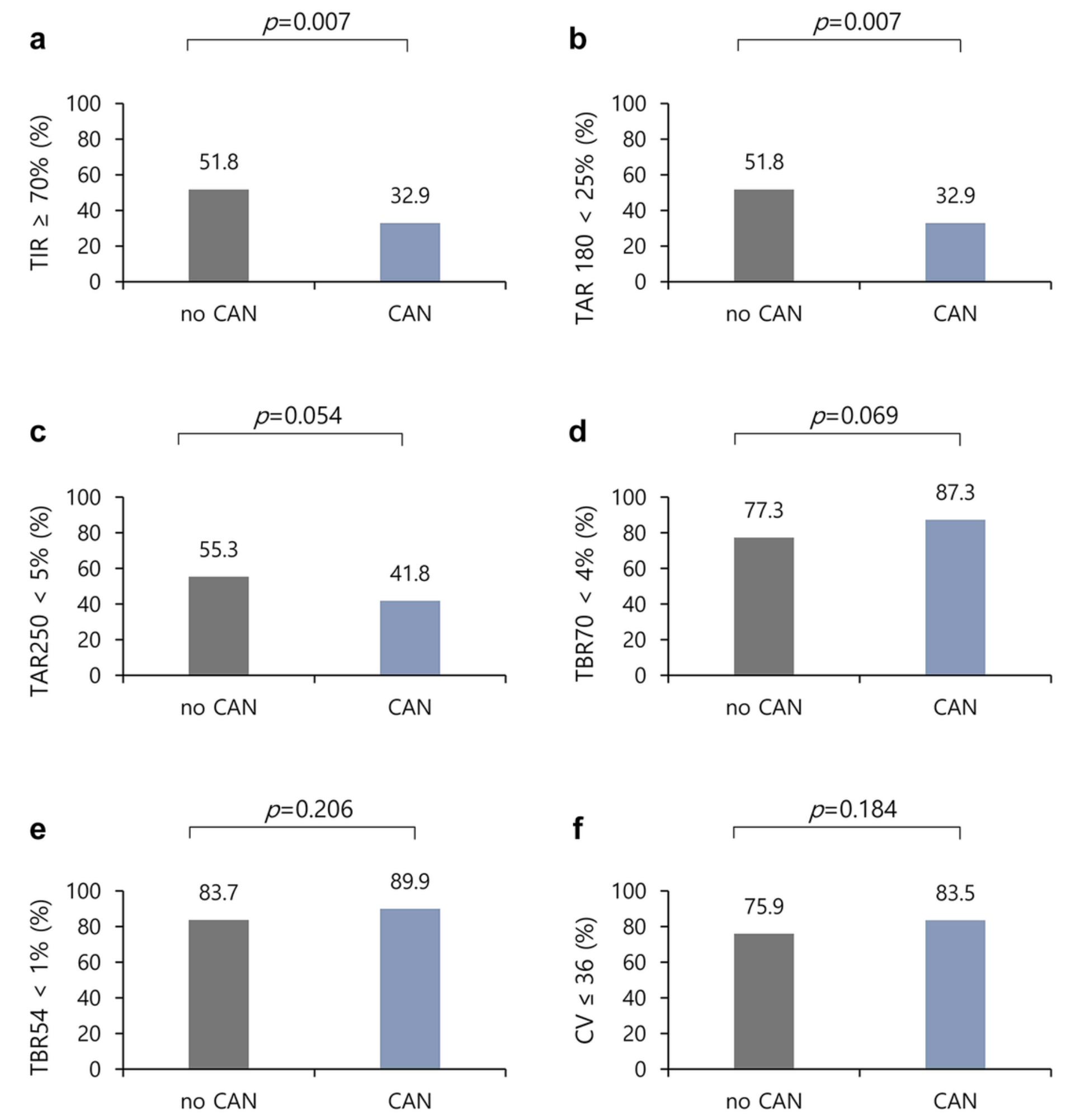

\section{Figure 2}

Prevalence of subjects who achieved the CGM targets according to the presence of CAN in study participants younger than 65 years $(n=220)$ CAN cardiovascular autonomic neuropathy, CGM continuous glucose monitoring, TAR time above range, TBR time below range, TIR time in range 

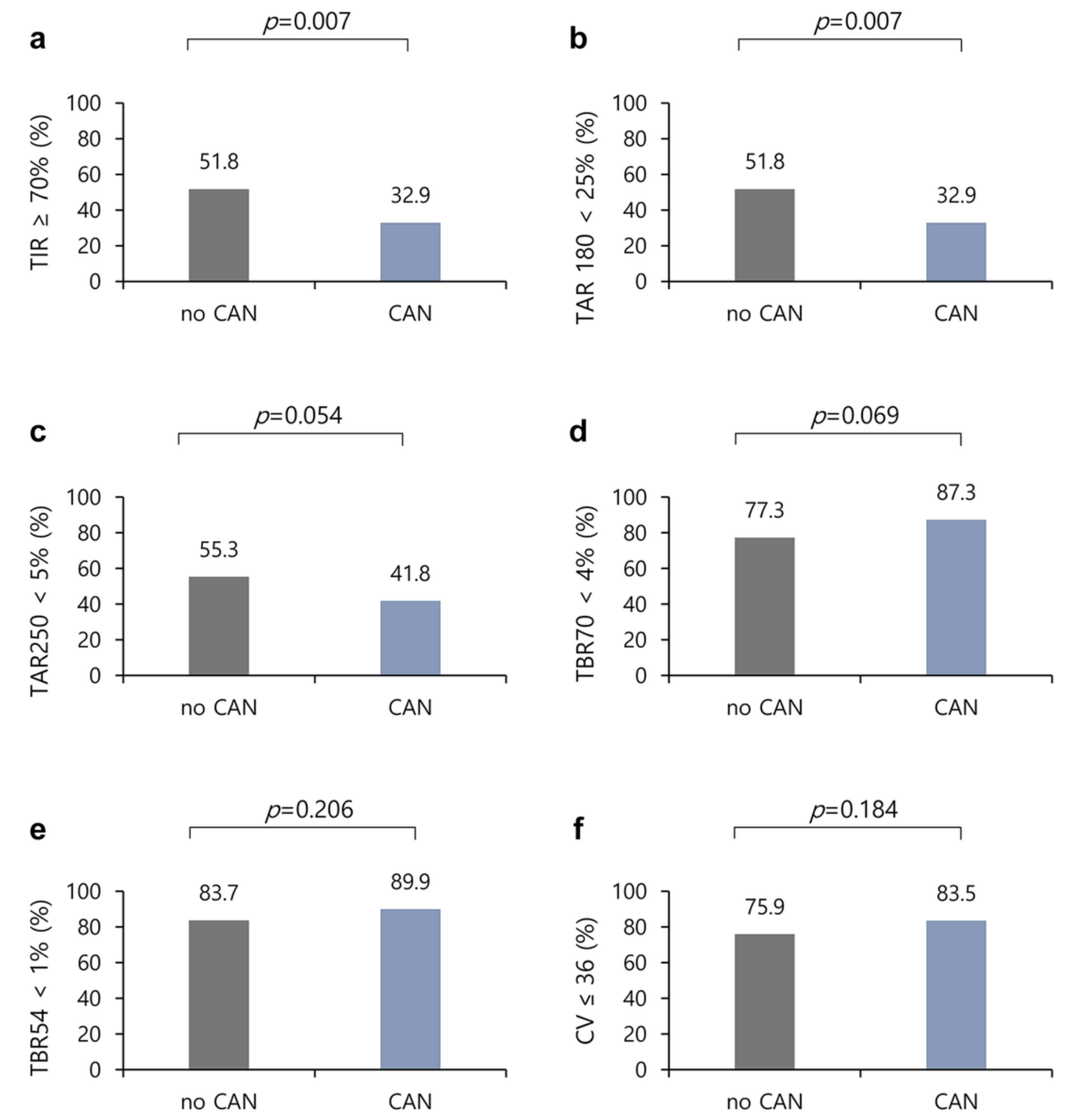

\section{Figure 2}

Prevalence of subjects who achieved the CGM targets according to the presence of CAN in study participants younger than 65 years $(n=220)$ CAN cardiovascular autonomic neuropathy, CGM continuous glucose monitoring, TAR time above range, TBR time below range, TIR time in range 


$$
\mathrm{p} \text { for trend }=0.001
$$

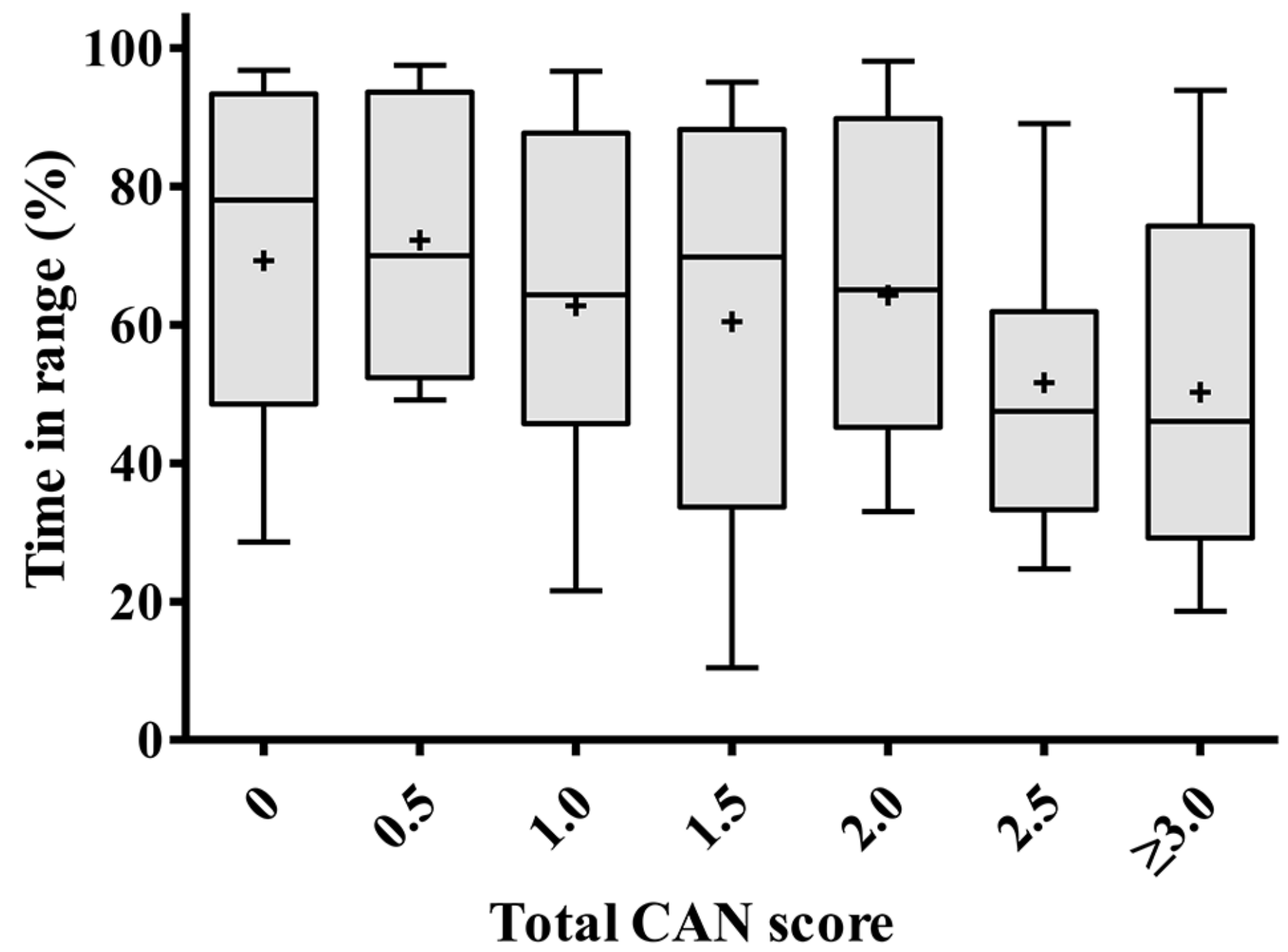

Figure 3

Boxplot of time in range according to total CAN score The box represents the upper and lower quartiles. Each + and horizontal line in the box indicates the mean and median value of time in range, respectively. The bar denotes 10-90 percentiles. CAN cardiovascular autonomic neuropathy, TIR time in range 


$$
\mathrm{p} \text { for trend }=0.001
$$

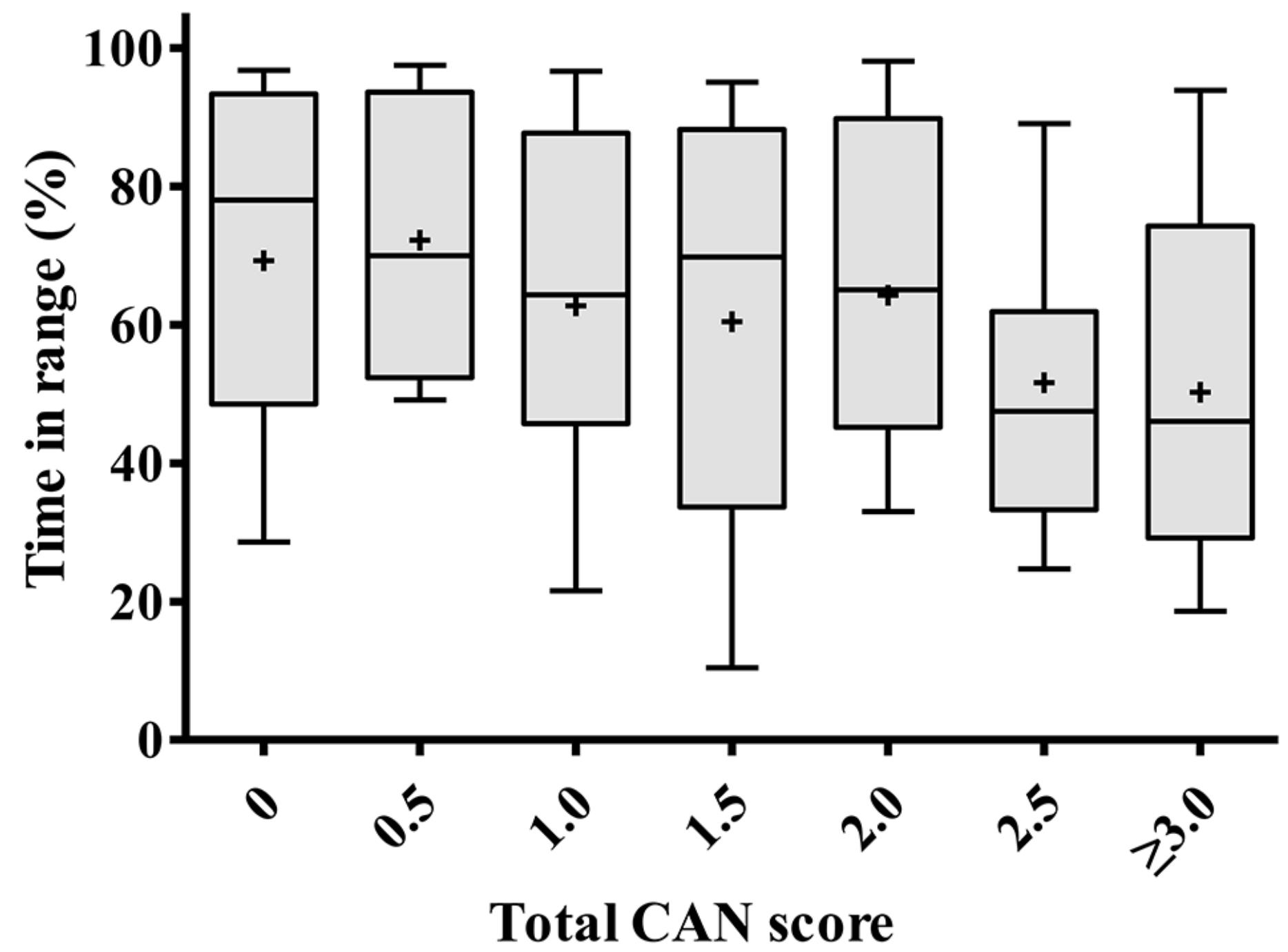

Figure 3

Boxplot of time in range according to total CAN score The box represents the upper and lower quartiles. Each + and horizontal line in the box indicates the mean and median value of time in range, respectively. The bar denotes 10-90 percentiles. CAN cardiovascular autonomic neuropathy, TIR time in range 


$$
\mathrm{p} \text { for trend }=0.001
$$

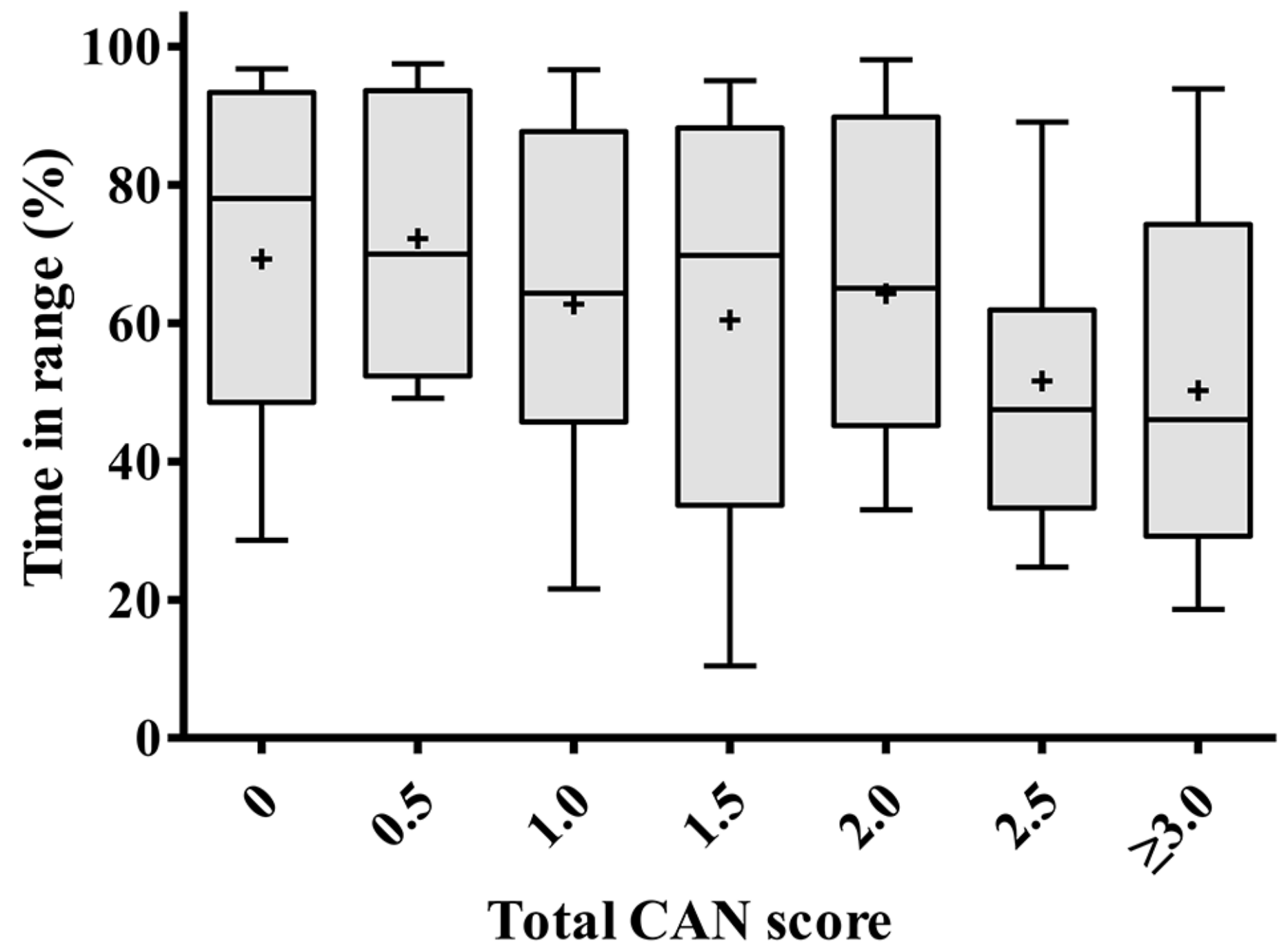

Figure 3

Boxplot of time in range according to total CAN score The box represents the upper and lower quartiles. Each + and horizontal line in the box indicates the mean and median value of time in range, respectively. The bar denotes 10-90 percentiles. CAN cardiovascular autonomic neuropathy, TIR time in range 